\title{
Study of the Quality of Barley Cleaning with Indented Cylinder from Grain Impurities
}

\author{
Nikolay Petrovich Tishaninov, Alexander Vitalyevich Anashkin, Haider Jameel Alshinayyin*, Konstantin \\ Nikolayevich Tishaninov
}

FSBSI, All-Russian Research Institute for the Use of Machinery and Petroleum Products in Agriculture, Tambov City 392022, Russian Federation

Corresponding Author Email: haiderjjsh2000@yahoo.com

https://doi.org/10.18280/ijdne.160311

Received: 4 March 2021

Accepted: 14 April 2021

\section{Keywords:}

grain mixture, impurity, indented cylinder, trier, separation degree

\begin{abstract}
Results of the study on the separation of crushed barley grains from the grain mixture along the length of the cell surface of indented cylinder, depending on the initial impurity concentration $\left(Z_{i}\right)$, the speed mode of the indented cylinder $(n)$ and the lifting angle of the upper edge of the front wall of the output tray $\left(\gamma_{\mathrm{n}}\right)$ relative to the horizon, are presented. The theoretical steadily decreasing trend in the intensity of the impurity component separation has been experimentally confirmed. The fact that crushed barley grains are a difficult-to-separate impurity has been established. When $n$ increases, the intensity of impurity separation increases dramatically. The $\gamma_{\mathrm{n}}$ angle increases the dependence of the process dynamics on the speed mode. At $\gamma_{n}=55^{\circ}$, the increase of $n$ from 40 to $50 \mathrm{rpm}$ increases the intensity of impurity separation from the grain mixture by 6.44 times. A decrease in the value of $\gamma_{\mathrm{n}}$ and an increase in the speed mode of operation lead to an increase in process losses. An increase in the initial contamination of the grain mixture under other equal operating conditions of the indented cylinder leads to a proportional increase in its residual contamination.
\end{abstract}

\section{INTRODUCTION}

The grain heap after harvesting is a multi-component mixture, which consists of seeds of the main crop, seeds of foreign cultivated and weed plants, and weeds of organic and mineral origin. The seed of the main crop may contain healthy seeds, damaged, shriveled, or empty, film-coated seeds. Defective grains of the main crop and grains of other crops are referred to as grain admixtures. Weed impurities include lumps of earth, pebbles, particles of ore and slag, parts of stems, awn, films, parts of leaves, seeds of wild (weed) plants, a harmful impurity. A weed admixture, especially of organic origin, is a favorable environment for the reproduction of harmful microorganisms, which leads to premature spoilage of grain during storage. Therefore, the freshly harvested grain heap must be immediately subjected to post-harvest cleaning and drying in case of high humidity.

The separation of impurity particles from the grain heap is based on the difference in their physical and mechanical properties with the grains of the main crop. This principle is the basis for the operation of various grain cleaning machines, separating the components according to the characteristics of the difference in the aerodynamic properties of particles, their sizes, shapes, surface properties, density, and electrical properties. In the presence of difficult-to-separate impurities in the grain mixture, comparable to the particles of the main culture in the thickness and width of the particles, separation is possible only with the help of cellular surfaces of the grains separating the grain mixtures along the length of the particles.

For efficient use of the indented cylinder in grain cleaning technologies, depending on the initial properties of the separated grain mixtures and their feed size, an optimal choice of operating modes and settings is required. The scientific literature has almost no studies of these relationships in relation to barley. Pavlovsky G.T. in the 60-70s of the last century studied the relationship between kinematic modes, the size of cell cylinders, the composition of grain mixtures and the efficiency of their cleaning process. As an efficiency criterion, the author suggests the ratio of the particles actually fallen into the tray to the theoretically possible amount. Despite all the uncertainty of calculation procedures of the "theoretically possible amount", the suggested criterion can be accepted, since in some cases the comparison base, if it is reproducible, is not so important for physical process evaluation. However, the suggested criterion does not allow a process dynamics assessment along the length of cell surface, which makes it impossible to work out objective solutions for process management. In his papers, Abiduev A.A. evaluates the process efficiency based on the analysis of the conditions of particle falling out of the cells and their discharge plume characteristics by the cell surface.

Based on the results of this analysis, the author suggests choosing the cell diameters and the angular position of the output tray for a specific speed mode of the indented cylinder. However, the author does not take into account the feed rate of the grain mixture into the cell cylinder and the initial properties of the grain mixture, which determine the completeness of the cell surface loading along the length, the impurity separation degree and residual contamination.

Danish scientists Ole Thomsen Buus, Johannes Ravn Jørgensen, and Jens Michael Carstensen investigated the process of particle fallout from the cells of a grating cylinder and their trajectories using high-speed video filming [1]. The authors have established regularity of the average values of the 
angle of precipitation of particles from the rotation speed of the indented cylinder for a simulated grain mixture, consisting of $50 \%$ whole and $50 \%$ crushed barley grains. However, in research, they used insufficient high-speed operating modes of the trier. The index of the kinematic mode was $\mathrm{k}=0.15-0.54$, while for commercially available triers and trier blocks $\mathrm{k}=$ $0.55-0.73$. Obviously, at these values of $k$, the range of angles of precipitation of particles and, possibly, the multimodality of their distribution will increase. The authors do not take into account the possibility of probabilistic capture of whole barley particles by cells and their trajectory. The unrealistic ratio of whole and crushed barley particles, as well as the uncertainty of the linear dimensions of crushed particles, does not allow identifying the research results with production conditions.

Romanian scientists [2-4] attempted to express the qualitative parameters of the working process of a cylindrical trier in the form of functions of its technological parameters and experimentally confirmed the validity of the developed mathematical model and the possibility of its application. They note that the quality of the selection of a short admixture depends not only on its initial content but also on the amount of grain mixture supplied, although no quantitative assessment is given.

Chinese scientists Meng et al. [5] studied the separation process of whole and crushed rice using a trier based on DEM modeling and a rice particle capture experiment. They found that with an increase in the number of cells, the angle of falling out of both whole and split rice particles increases. The optimal angle of installation of the receiving chute was set by the authors at the level of $60^{\circ}$, which ensures the separation efficiency at the level of $80 \%$.

Korean authors, Kim and Park [6] investigated the efficiency of the process of separating broken rice using a laboratory trier. They found that the angular position of the receiving chute and the rotation speed of the cylinder has a statistically significant effect on the separation efficiency of the components under study. In this case, the shape of the cell had practically no effect on the separation efficiency. For an industrial design of a trier for separating split rice, the authors recommend a hemispherical cell, the size of which should be chosen slightly larger than the actual size of the separated particles.

However, in their studies, the authors [2-6] did not use barley-based grain mixtures, which limits the possibility of using their results in its trier cleaning.

Researches of FSBSI scientists "All-Russian Research Institute for Use of Machinery and Petroleum Products in Agriculture" [7-9] confirmed the importance of interrelationships between the operating and tuning parameters of the trier and the quality indicators of the grain cleaning process, their importance for developing solutions for process control. The patterns of intensity changes of particle separation and the cell filling degree with the main crop grains along the cell surface length has been established, which is required to justify the setting parameters based on trade-off factors of the quality of indented cylinder operation [10-12].

In these studies, the main crop of the grain mixture was wheat, and grain mixtures with grain admixture were not considered. Therefore, the increase of the knowledge base concerning the impurity components composition and types of the main crop in grain mixtures is a relevant objective and requires additional research on the process of grading barley cleaning from grain impurities.

\section{MATERIALS AND METHODS}

The followings are used in the paper: a device for cell surface study [13-16], which replaces the spatial model of the study of cell surfaces with a temporary one; a performance identification method of indented cylinders based on the bench test results [17]; a grid classifier; electronic scales.

The device contains an indented cylinder with a diameter of $300 \mathrm{~mm}$ and a length of $300 \mathrm{~mm}$. The indented diameter is 5 $\mathrm{mm}$. The structural and process diagram of the device for cell surface studies is shown in Figure 1, its general appearance in Figure 2.

The device operates as follows. The grain mixture 7 is unloaded into the cell cylinder 1 by means of a funnel and leveled along it. The drive disc 3 is switched on. Impurity particles from the layer in contact with the cell surface are picked by the cells and fed into tray 2 attached by the end wall 10 to the frame 9 bracket 6 .

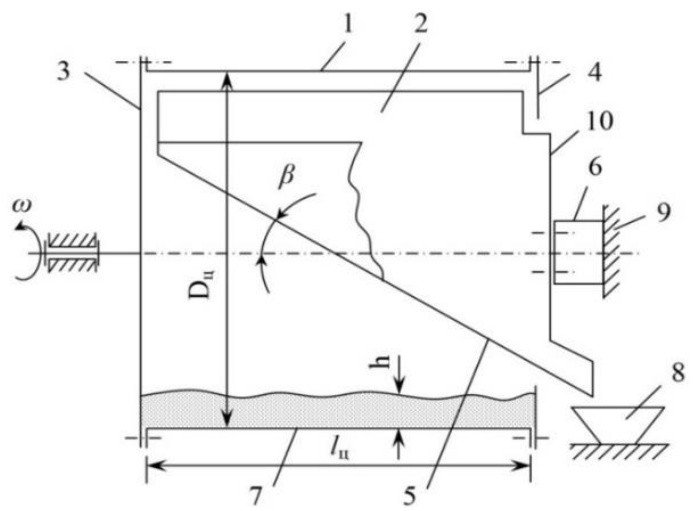

1 - cell cylinder; 2 - tray; 3 - drive disk; 4 - locking ring; 5 - inclined tray bottom; 6 - bracket; 7 - grain mixture; 8 - container; 9 - frame; 10 - end wall of the tray

Figure 1. Diagram of the device for cell surfaces study

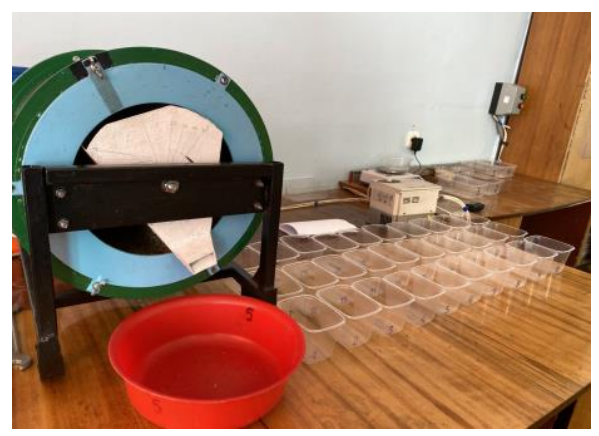

Figure 2. General appearance of the device for cell surface study

Having an initial kinetic energy, the impurity particles roll down the inclined bottom 5 of the tray 2 into containers 8 that change at specified intervals.

At the end of the process, which is controlled according to the scheduled time, the drive disc is turned off, the blocking ring 4 is removed and the main crop kernels are unloaded into an additional container by rolling over.

Due to the installation of the blocking ring 4 (Figure 1) and the use of tray 2, which ensures continuous removal of the emitted grains, a cyclic mode of operation of the device is ensured. This allows the process to be investigated for an unlimited amount of time until the complete isolation of the impurity component. With the help of the method developed 
by us for identifying the results of bench studies, it became possible to compare the data obtained with the operation of a trier with a cylinder of any diameter and unlimited length.

The temporary study model of the components separation processes of grain mixtures by cell surfaces, together with the performance identification method of indented cylinders based on the bench test results, provides results that are invariant with respect to the indented cylinder length. With the use of the presented equipment, for the first time, we obtained the regularities of the allocation of short impurities for cellular surfaces, equivalent to indented cylinders $12-18 \mathrm{~m}$ long. It is possible to design and manufacture such a trier for research, but the costs for it will be incommensurate with our device. In this way. At the same time, the research costs were significantly reduced without compromising the reliability of the results.

\section{RESULTS AND DISCUSSION}

A grain mixture based on barley, including a grain impurity in the form of crushed kernels of the main crop, was used in the study. The physical and mechanical properties of the impurity component coincide with the particle's properties of the main crop, except for the particle length. The average length of crushed kernels was $5.1 \mathrm{~mm}$, and the length of barley kernels was $10.1 \mathrm{~mm}$.

This grain mixture option is unique - the intensity of the impurity component separation does not depend on its dynamic separation in the circulating layers of the segment, since it does not exist - there is no "settlement" and no "floating-up" [18], in Figure 3.

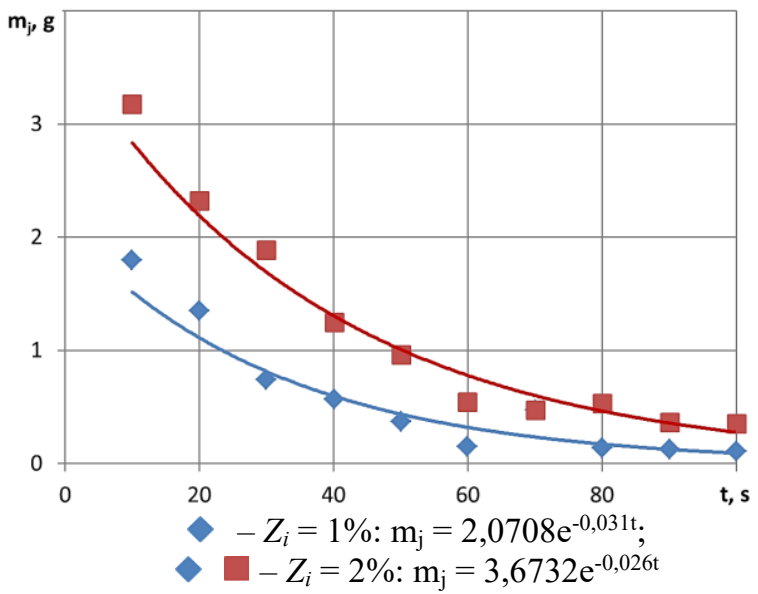

Figure 3. Dynamics of crushed barley particles separation from the grain mixture when $n=40 \mathrm{rpm}$ and $\gamma_{\mathrm{n}}=35^{\circ}$

The intensity dynamics of the crushed barley grains separation, shown in Figure 3, is close to the theoretical, steadily decreasing one, when the pattern is obtained from the following conditions: impurity particles in the circulating layers are distributed evenly; the content of impurity particles in the circulating segment decreases with decreasing intensity; the impurity particles located in the contact layer are completely segregated in one cycle due to the multiple excess of the separating effects in the cockle cylinder relative to the actual amount of impurity particles in the contact layer.

For the formulated conditions for the analytical evaluation of the impurity particles separation dynamics from the grain mixture, the following equation is valid:

$$
Z_{j}=Z_{i}\left(1-\sum_{1}^{j=k} n_{a j} / P\right)
$$

where,

$Z_{j}$ is the segment contamination after the $j$-th cycle of impurity separation, $\%$;

$Z_{i}$ - initial contamination of the grain mixture, $\%$;

$k$ - number of separation cycles, pcs;

$n_{a j}$-number of impurity particles separated in thej-th cycle, pcs;

$P$ - initial number of impurity particles in the segment, pcs.

By analogy with Eq. (1), for the process of impurity particles separation with an interval estimate (time-wise) in the $i$-th interval of separated masses, the following formula can be written:

$$
Z_{j}=Z_{i}\left(1-\sum_{1}^{j=k} m_{j} / M\right)
$$

where, $m_{j}$ is the mass of impurities separated from the segment in the $j$-th time interval, g.

$M$ - initial mass of the impurity in the segment, $\mathrm{g}$.

Analysis of the crushed barley grains separation dynamics according to formula (2) allowed to establish that the residual contamination of the grain mixture even after 100 seconds, which is 1.8 times longer than the process duration in a cockle cylinder, is $0.42 \%$. This is higher than the accepted values and when $Z_{i}=1 \%$. At the length of the standard cell cylinder, the residual contamination is even higher $\left(R_{d}=0.504 \%\right)$ with the separation degree $D_{r s}=49.6 \%$.

The procedure for calculating the process indicators for the standard length of the cellular cylinder, according to the method developed by Tishaninov and Anashkin [19], is as follows. According to the relationship of the average speed of the axial displacement of the grain segment in the cellular cylinder with the amount of grain mixture feeding into it, the time during which the segment is displaced by an amount equal to the working length of the cellular cylinder in the stand is determined; further, according to the time scale for each detected intensity of the release, the number of time intervals of measurements is determined; the average interval value of the selection intensity is determined; the obtained values are added up. For the corresponding moment of the process, the degree of impurity release (Drs) and residual contamination (Rd) of the grain mixture, corresponding to the length of a standard grating cylinder, are determined from the sum of the separated impurity particles.

Cleaning the grain mixture with crushed barley grains with $Z_{i}=2 \%$ provides comparable values of the impurity separation degree in terms of process time in the cockle cylinder of standard indented cylinder $-D_{r s}=48 \%$, at the end of the period $t=100 \mathrm{~s}$, the separation degree of $D_{r} \approx 58 \%$ in both options of the initial contamination - in the first $D_{r}=58.4 \%$, and in the second $D_{r}=57.9 \%$. However, the residual contamination of the grain mixture when $Z_{i}=2 \%$ is $1.04 \%$, which is approximately twice as high as in the first case - when $Z_{i}=1 \%$.

This is the fundamental difference between grain mixtures with crushed particles of the main crop and grain mixtures with easily separated impurities (seeds of mustard, millet, sorghum, vetch) [20]. In the first case, there is no effect of dynamic separation and the impurity separation process is slow, and in the compared options, the initial concentration of impurity components $\left(Z_{i}\right)$ determines the potential of dynamic 
separation and the duration of their separation, which has little to do with the $Z_{i}$ value.

The evaluation of the process dynamics of crushed barley grains separation from the grain mixture over time periods (55 and $100 \mathrm{~s}$ ) confirmed the comparability of the $D_{r s}$ and $D_{r}$ values, regardless of $Z_{i}$. However, as you can see in Figure 3, at the initial measurement time intervals, the ratio of the absolute values of the separated impurity masses is $<2$. This is due to the predominance of the "eating out" effect of impurity particles from the cells at the specified time intervals of the process when $Z_{i}=2 \%$ in comparison with the conditions when $Z_{i}=1 \%$.

The relationship between the crushed barley grains separation dynamics from the main crop and the operation speed mode is shown in Figure 4.

As you can see in Figure 4, the mass gain of the separated impurity component from the grain mixture for the first-time interval $(t=10 \mathrm{~s})$ with an increase in $n$ from 40 to $55 \mathrm{rpm}$ was $241 \%$. In subsequent time intervals of the process, the separated masses ratio at different speed modes of operation is equalized.

There is a general decreasing relationship between $m_{j}=f(t)$ for all speed modes. A significant difference of separated masses in the first interval with a maximum increase in speed mode of operation is due to the increased discharge plume coverage of impurity particles by the output tray. However, even when $n=55 \mathrm{rpm}$ and $\gamma_{n}=35^{\circ}$, only $89 \%$ of the impurity particles are separated from the grain mixture in $t=100 \mathrm{~s}$.

This is because the average length of the impurity particles exceeds the size of the cells. This increases the likelihood of early loss of particles from the cells and extension of the coverage angle of their discharge plume, and the effect of inertial particle retention with increasing $n$ does not make it possible to compensate for the discharge plume extension due to their linear size.

When $\gamma_{n}$ is increased to $55^{\circ}$, you have even more contras dynamics of the crushed barley grains separation from the grain mixture, Figure 5.

An increase in the speed mode from $40 \mathrm{rpm}$ to $50 \mathrm{rpm}$ leads to an increase in the mass of the separated impurity particles in the first-time interval $t=20$ seconds by 6.44 times (from 0,86 to $5,54 \mathrm{~g}$ ). This is due to the fact that when $\gamma_{n}=55^{\circ}$ and $n=40 \mathrm{rpm}$, the output tray picks up only the upper part of the particle discharge plume by the cells - a small part in terms of coverage angle.

It is obvious that the speed mode of operation $n=40 \mathrm{rpm}$ when $\gamma_{n}=55^{\circ}$ is not sufficient. Therefore, only $7.1 \%$ of the impurity mass is separated at the standard length of the cell cylinder. In $t=180$ seconds, which is equivalent to the process flow in the cell cylinder with a length of $7.2 \mathrm{~m}, 24.6 \%$ of the impurities are separated. With an increase in $n$ to $50 \mathrm{rpm}$, the impurity separation degree at the standard length $(l=2.2 \mathrm{~m})$ of the cell cylinder increased not significantly. It was $20.4 \%$ at $R_{d s}=1.593 \%$, which does not meet the requirements for the process quality.

It should be noted that the bench speed mode of operation $n$ $=40 \mathrm{rpm}$ corresponds to the full-scale cell cylinder operating mode $n_{l}=28.3 \mathrm{rpm}$ and the kinematic mode coefficient $k_{l}=$ 0.268. This is the lower limit of the speed mode of the fullscale cylinder operation. However, even at $n=50 \mathrm{rpm}\left(n_{3}=\right.$ $\left.35.4 \mathrm{rpm} ; k_{3}=0.419\right)$, the required degree of crushed barley grains separation is also not achieved. A further increase in the speed mode of operation leads to an increase in process losses.

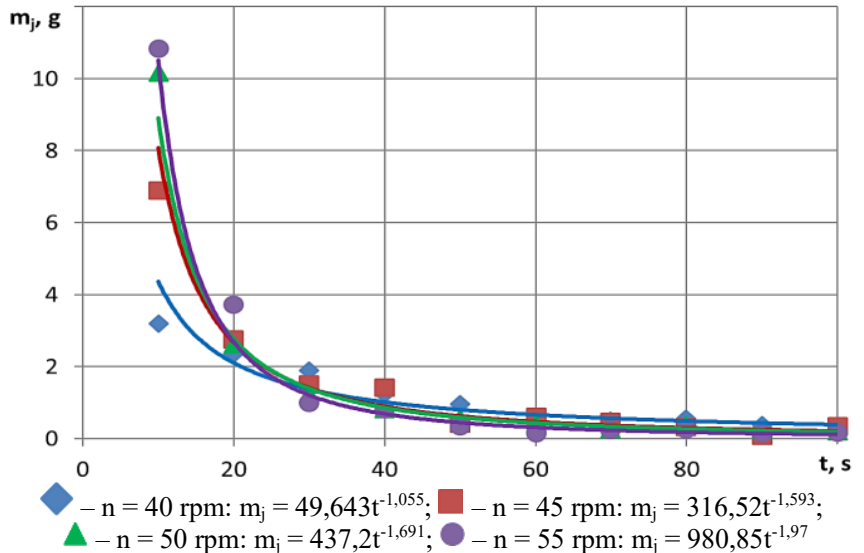

Figure 4. The relationship between the crushed barley grains separation dynamics $\left(\mathrm{m}_{\mathrm{j}}\right)$ from the main crop and the rotation speed $(n)$ of the cell drum at $\gamma_{\mathrm{n}}=35^{\circ} ; m=1000 \mathrm{~g} ; Z_{i}=2 \%$

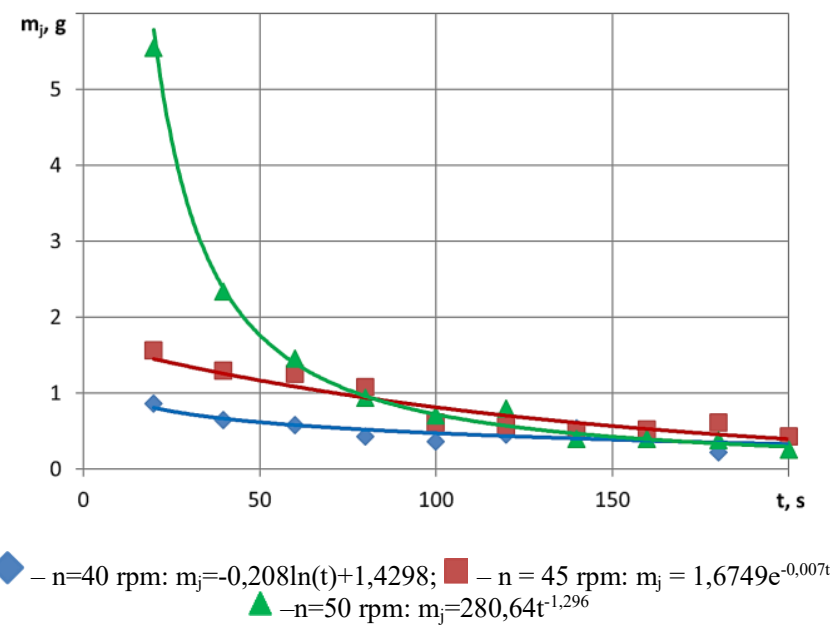

Figure 5. The relationship between the crushed barley grains separation dynamics $\left(\mathrm{m}_{\mathrm{j}}\right)$ from the main crop and the rotation speed $(n)$ of the cell drum at $\gamma_{n}=55^{\circ} ; m=1000 \mathrm{~g} ; Z_{i}=2 \%$

\section{CONCLUSIONS}

Grain impurity in the form of crushed grains of the main crop cannot be separated from grain mixture without the use of trier cleaning. It is been established that the dynamics of the intensity of trier separation of crushed barley grains from the grain mixture is equally decreasing. It is not subjected to dynamic segregation in the circulating layers of the segment inside the indent cylinder. The speed mode of the trier and the angular position of the output tray have a significant influence on the intensity of the separation of crushed barley grains. An increase in the speed mode of operation and a decrease in the angle of lifting of the working edge of the output tray led to an increase in technological losses of grain of the main crop. The amount of residual contamination directly depends on the initial content of the impurity component in the grain mixture before cleaning. Crushed barley grains are difficult to separate the impurity, the main reason for which is their unstable placement in the cells of the doll cylinder. To separate it, you need a block of doll cylinders. Further studies of the barley cleaning by using indented cylinder (Trier) from grain impurities comparable in transverse dimensions with the particles of the main crop should be carried out under 
production conditions on grading blocks as part of the grain cleaning technology.

\section{REFERENCES}

[1] Buus, O.T., Jørgensen, J.R., Carstensen, J.M. (2011). Analysis of seed sorting process by estimation of seed motion trajectories. In: Heyden A., Kahl F. (eds) Image Analysis. SCIA 2011. Lecture Notes in Computer Science, vol 6688. Springer, Berlin, Heidelberg. https://doi.org/10.1007/978-3-642-21227-7 26

[2] Sorica, C., Pirna, I., Bracacescu, C., Marin, E., Postelnicu, E. (2012). Cinematic analysis of particle of impurity in conditioning process of grains into indented cylinder separators. 11-th International Scientific Conference. Engineering for Rural Development, Jelgava, LATVIA. pp.

60-66.

http://www.tf.llu.lv/conference/proceedings2012/Papers /010_Sorica_C.pdf.

[3] Sorică, C., Pirnă, I., Găgeanu, P., Marin, E., Postelnicu, E. (2011). Indented cylinder separators - quality characteristics expressed as functions of process parameters. Proceedings of International Conference on Innovations, Recent Trends and Challenges in Mechatronics, Mechanical Engineering Aand New HighTech Products Development - MECAHITECH'11., 3: 341-347.

https://www.incdmtm.ro/mecahitech2011/articole/Pp34 1-347.pdf.

[4] Sorică, C., Bracăcescu, C., Manea, D., Sorică, E., Epure, M., Covaliu, C. (2014). Considerations regarding the optimization of the mechanical conditioning process of grain into the indented cylinder separators. ANNALS of Faculty Engineering Hunedoara - International Journal of Engineering, pp. 335-338. http://annals.fih.upt.ro/pdffull/2014/ANNALS-2014-3-52.pdf.

[5] Meng, X.Y, Jia, F.G., Qiu, H.L., Han, Y.L., Zeng, Y., Xiao, Y.W., Chen, P.Y. (2019). DEM study of white rice separation in an indented cylinder separator. Powder Technology, 348:

$1-12$. https://doi.org/10.1016/j.powtec.2019.03.013

[6] Kim, M.H., Park, S.J. (2013). Analysis of broken rice separation efficiency of a laboratory indented cylinder separator. Journal of Biosystems Engineering, 38(2): 95102. http://dx.doi.org/10.5307/JBE.2013.38.2.095

[7] Tishaninov, N.P. (2020). Automatically-controlled speed mode control of the indented cylinder operation. Science in Central Russia, (5): 24-31.

[8] Tishaninov, N.P., Anashkin, A.V. (2018). Control of the flow characteristics of the indented cylinder by the position of output tray. Science in Central Russia, (2): 28-34.

[9] Tishaninov, N.P., Anashkin, A.V. (2018). Analysis of indented cylinder performance indicators based on the results of bench tests. Science in Central Russia, (1): 27 34.

[10] Tishaninov, N.P., Anashkin, A.V. (2019). The separation intensity of the main crop grains from grain mixtures with long impurities. Vestnik of the APK
Verkhnevolzhya, (3): 74-77.

[11] Anashkin, A.V., Tishaninov, N.P. (2020). The relationship between the cleaning of the wheat by an indented cylinder with the concentration of long impurities. Science in Central Russia, (3): 60-67.

[12] Tishaninov, N.P., Anashkin, A.V. (2013). Results of studies of the grain mixture separation process with the indented cylinder // Science in Central Rus-sia, (6): 3745.

[13] Tishaninov, N.P., Anashkin, A.V., Alshinayyin, H.J.J. (2017). Justification of the optimal parameters and device operation modes for separating grain mixtures along the particle length. Science in Central Russia, (1): 10-19.

[14] Tishaninov, N.P., Anashkin, A.V., Al'shinayyin, K.D. (2016). Justification of operating modes and parameters of the device for sieving grain mixes samples. Science in the central Russia, (3): 74-80.

[15] Patent of the Russian Federation No. 2616201. Russian Federation. Test bench for cell surfaces/Tishaninov N.P., Anashkin A.V. - No. 2016108182; application 09.03.2016; published on 13.04.2017, Gazette No. 11.

[16] Patent of the Russian Federation No. 2647526. Russian Federation. Device for separation of impurities from grain mixtures / N.P. Tishaninov, A.V. Anash-kin. - No. 2017114302; application 24.04.2017, published on 16.03.2018, Gazette No. 8.

[17] Tishaninov, N.P., Anashkin A.V. (2018). Justification of the identification method of indented cylinder performance based on the results of bench tests. Science in Central Russia, (1): 20-27.

[18] Tishaninov, N.P., Anashkin, A.V. (2017). Scientific research of dynamic segregation of impurities during allocation of wheat millet. Science in the central Russia. (2): 69-77.

[19] Tishaninov, N.P., Anashkin, A.V. (2015). Theoretical analysis of the dynamics of the release of short impurities from grain mixtures by a cellular surface. Science in Central Russia, (1): 46-58.

[20] Tishaninov, N.P., Anashkin, A.V. (2017). Separation quality of mustard and sorghum seeds from wheat by indents surfaces. Science in Central Russia, (3): 42-48.

\section{NOMENCLATURE}

$\mathrm{Zi} \quad$ initial impurity concentration

$\mathrm{n} \quad$ speed mode of the indented cylinder

$\gamma \mathrm{n} \quad$ the lifting angle of the upper edge of the front wall

$\mathrm{D}_{\mathrm{rs}} \quad$ the impurity separation degree on the standard length of the cell cylinder

the mass

time

number of separation cycles, pcs

number of impurity particles separated in the $j$ - th cycle, pcs

initial number of impurity particles in the segment, pcs

$M \quad$ initial mass of the impurity in the segment, $g$.

$R_{d} \quad$ the residual contamination 